\title{
Comparison of the toxicity of aqueous and ethanol fractions of Angelica keiskei leaf using the eye irritancy test
}

\author{
HYEONG-U SON $^{1 *}$, EUN-KYUNG YOON ${ }^{1 *}$, YONG-SOO CHA ${ }^{1}$, MIN-A KIM $^{1}$, \\ YONG-KYU SHIN ${ }^{2}$, JONG-MYUNG KIM ${ }^{2}$, YONG-HEE CHOI ${ }^{1,3}$ and SANG-HAN LEE ${ }^{1,3}$ \\ ${ }^{1}$ Department of Food Science and Biotechnology, Graduate School, Kyungpook National University, \\ Daegu 702-701; ${ }^{2}$ Bion Co. Ltd., Techno Building, Daegu 702-842; ${ }^{3}$ Food and Bio-Industry \\ Research Institute, Kyungpook National University, Daegu 702-701, Republic of Korea
}

Received April 5, 2012; Accepted August 8, 2012

DOI: $10.3892 /$ etm.2012.677

\begin{abstract}
To determine whether aqueous and ethanol fractions of the Angelica keiskei leaf exert toxicity when used for cosmetic purposes, we performed the acute eye irritancy test. Animals were treated with sample fractions (100 mg/dose) according to standard procedure guidelines. No significant changes or damage was detected in the fraction-treated groups in terms of ocular lesions in the cornea, the size of the cornea with turbidity, swelling of the eyelid and emission discharge. However, sodium dioctyl sulfosuccinate, a positive control, induced severe toxic symptoms. Thus, aqueous and ethanol fractions of Angelica keiskei do not appear to induce acute toxicity in the eye lens, as assessed from anatomical and pathological observations in the rabbit eye. Our results collectively suggest that aqueous and ethanol fractions show promise as cosmetic ingredients that do not cause eye toxicity.
\end{abstract}

\section{Introduction}

Many medicinal plants provide beneficial sources of vitamins, dietary fiber and phytochemicals. A number of herbs are widely used owing to their flavor and healthy constituents which, not only regulate body homeostasis, but also prevent degenerative diseases (1-3). Angelica sp. is a common vegetable known for its vitamin content (4). Early studies have shown that the vegetable contains vitamins $C, B 1$ and B2, chlorophylls and diverse minerals $(4,5)$. The moist leaves exhibit exceptional antioxidant activity and therefore the herb is widely used as a functional food ingredient. Additionally,

Correspondence to: Professor Sang-Han Lee, Department of Food Science and Biotechnology, Graduate School, Kyungpook National University, Daegu 702-701, Republic of Korea

E-mail: sang@knu.ac.kr

*Contributed equally

Key words: Angelica keiskei, eye irritancy test, fraction, cosmetic ingredient the herb has therapeutic effects on hypertension, constipation, diuresis, arteriosclerosis and most importantly cancer, which are attributable to its small molecular constituents, such as flavonoids, saponin and coumarins (5). Further research on the chemical composition of oils has led to the identification of dozens of small compounds (6). The oils exhibit strong activity in suppressing PANC-1 pancreatic cancer cell and Crl breast cancer cell growth (7). Angelica keiskei is broadly employed in alternative medicine as a remedy to treat bowel disturbance syndrome, arthritis and immune diseases (8). However, there is limited scientific information regarding its effects on various degenerative disorders. Findings of another study have shown that Angelica keiskei has the ability to reduce inflammation in a chronic ethanol-induced in vivo test. Upon treatment of ICR mice with 10,25 and $50 \mathrm{mg} / \mathrm{kg}$ extracts p.o., alcohol-induced hepatotoxicity was improved, indicating that Angelica keiskei indirectly safeguards the liver against oxidative stress induced by free radicals (9). An earlier investigation by our group revealed anti-asthmatic activities of an aqueous leaf extract in an ovalbumin-induced animal model (10). Mice sensitized to ovalbumin were orally administered the Angelica archangelica extract and their lungs were analyzed via hematoxylin and eosin (H\&E) staining to measure the IL-4/13 cytokine content. The extract exhibited strong anti-asthmatic activity via regulation of the $\mathrm{CD} 4^{+}$cell population, IL-4/13 expression and asthma-related molecular markers in the lungs (10).

The Korea Food and Drug Administration has recommended submission of data from eye irritancy, skin irritancy and phototoxicity tests to obtain approval and authorization of the use of test compounds as functional cosmetic ingredients(s). Plant extracts with pungent flavors appear to cause irritation when exposed to the skin (4). Unwanted mild or transient reactions to cosmetics are common in patients with allergic contact dermatitis. Various adverse effects including acute/chronic toxicity, irritation and sensitization, can be assessed using in vivo, in vitro, semi in vivo and ex vivo animal models, even in modified tests (11-13). A specific component or constituent should not exert toxic effects on the skin (particularly the eye in the case of cosmetics) and should only be passed and approved in cases where no damage/changes to the eye lens are observed in animals or clinical trials for the development of cosmetics (14). Although cosmetic ingredients rarely trigger 
A

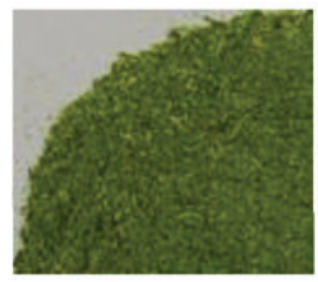

B

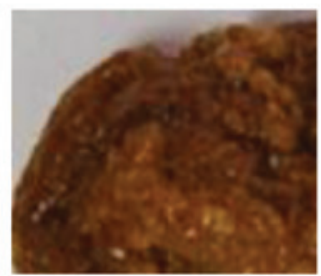

C

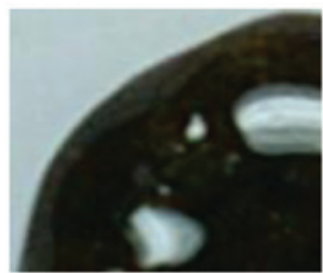

D

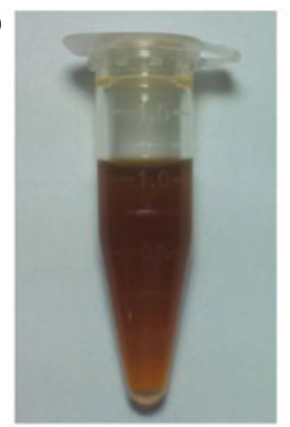

$\mathbf{E}$

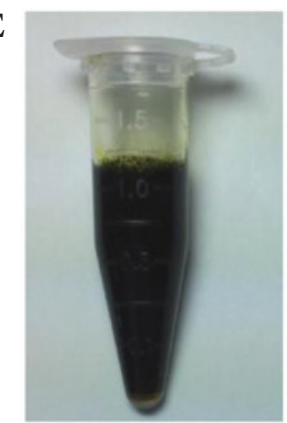

Figure 1. Images of the aqueous and ethanol fractions of Angelica keiskei leaf are shown. (A) Slice-dried leaf was pulverized and extracted with (B) distilled water or (C) ethanol at $60^{\circ} \mathrm{C}$ for $12 \mathrm{~h}$ in a shaking incubator and filtered. Lyophilization was performed to obtain the final sample powder. The (D) aqueous and (E) ethanol fractions (each $100 \mathrm{mg} / \mathrm{ml}$ ) are shown.

serious damage, their advantages in skin protection or tissue regeneration should be emphasized.

In the current study, we performed the acute eye lens mucosal irritation test with Angelica keiskei leaf fractions using an in vivo animal model. Various parameters were assessed by comparing the degree of acute toxicity induced to confirm whether these fractions have potential for development in cosmetic applications.

\section{Materials and methods}

Animals and care. New Zealand White rabbits ( 9 weeks old, male, weighing 2.0-2.2 kg) were purchased from M\&J Animal Supplies Co. (Seoul, Korea) and fed a commercial diet (Purina, Seoul, Korea) and water ad libitum. Animal protocols were performed in accordance with the guidelines of the Committee of International Association for the Study of Pain on Research and Ethical Issues (15). The rabbits were allowed to adjust to the laboratory surroundings for at least 1 week prior to the experiments. The number of rabbits in each group was 3 .

Sample preparation. Angelica keiskei leaf purchased from Myung-il Farm Co. (Eumsung, Korea) was used throughout the experiments. The slice-dried leaf was pulverized with a homogenizer (20,000 rpm for $15 \mathrm{~min}$; Shinil, Seoul, Korea). Powder was extracted with distilled water (powder:distilled water $=1: 10 ; \mathrm{w} / \mathrm{v})$ or ethanol $($ powder:50\% ethanol $=1: 6 ; \mathrm{w} / \mathrm{v})$ at $60^{\circ} \mathrm{C}$ for $12 \mathrm{~h}$ in a shaking incubator (JSR, Gongju, Korea), followed by filtration (Filter paper No. 1, Whatman, Schleicher \& Schuell, Buckinghamshire, UK). Lyophilization was carried out in a freeze-dryer (Bondiro, Il-Shin, Seoul, Korea) to obtain the final samples (Fig. 1B and C). The voucher specimens of the Angelica keiskei leaf and powder were deposited in the Laboratory of Food Enzyme Biotechnology, Kyungpook National University (\#2010-Ak).

Draize eye irritancy test. Fractions $(100 \mathrm{mg} / \mathrm{ml}$ of concentrated aqueous and ethanol fractions) were dripped into the eyes of each New Zealand White rabbit $(n=5)$ with their eyes held open and clipped at the lid. As a positive control, 10\% of sodium dioctyl sulfosuccinate was employed. Progressive damage/changes to the rabbit eye were recorded each day for 7 days. Reactions to the fractions included swelling of the eyelid,

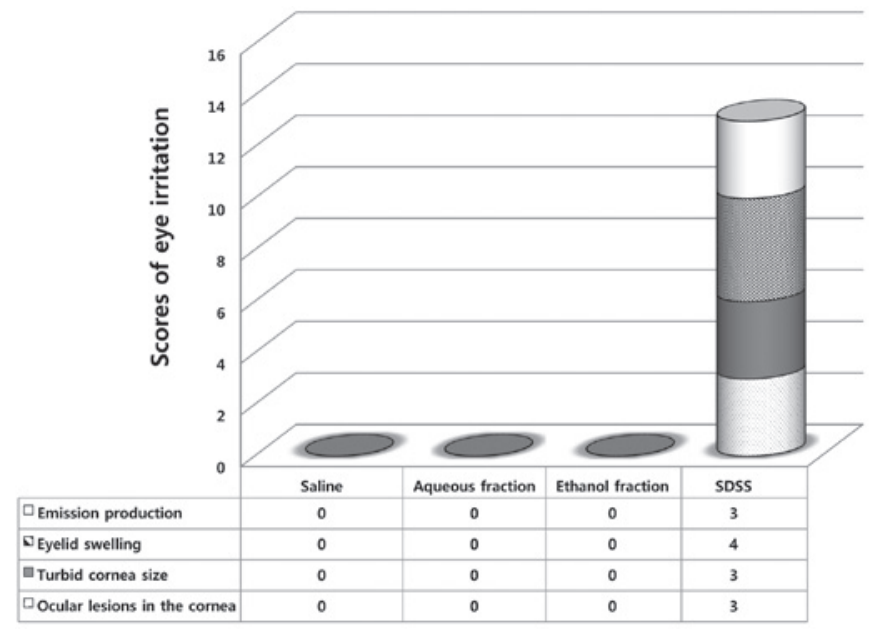

Figure 2. Comparison of the aqueous and ethanol fractions of the Angelica keiskei leaf in an acute eye lens mucosal irritancy test. Final scores of acute eye lens mucosal irritancy are shown. Each score is the sum of the total degree. Data denote a classical result of five independent observations. Dotted, ocular lesions in the cornea; black, turbid cornea size; slashed, eyelid swelling; white, emission production. SDSS, sodium dioctyl sulfosuccinate.

iris inflammation, ulceration and hemorrhaging, as described in other studies, with slight modifications $(16,17)$.

Sample treatment. Following instillation of the aqueous and ethanol fractions of the Angelica keiskei leaf, eye lens mucosa was evaluated for local mucosal irritation. Saline was used as the control. The conjunctival sac in the right eye of the rabbits was treated with the sample (Fig. 1D and E; concentrated to $0.1 \mathrm{ml}$ ), control (saline) or positive control (sodium dioctyl sulfosuccinate). After applying once for $2 \mathrm{sec}$, washing with saline was performed as for the control group. The undiluted sample was administered once under the eyelid, which was slightly pulled away to form a space to allow easy administration (aqueous or ethanol fraction, $0.1 \mathrm{ml}$ each) into the conjunctival sac. Following this, the cornea, iris and conjunctiva were examined daily at the designated times $(1,2,3,4,5$, and 7 days) to evaluate acute toxicity in the eye lens mucosa.

Analysis of parameters. Lesions were observed in experiments whereby the test substance was not applied to the left eye for 
A

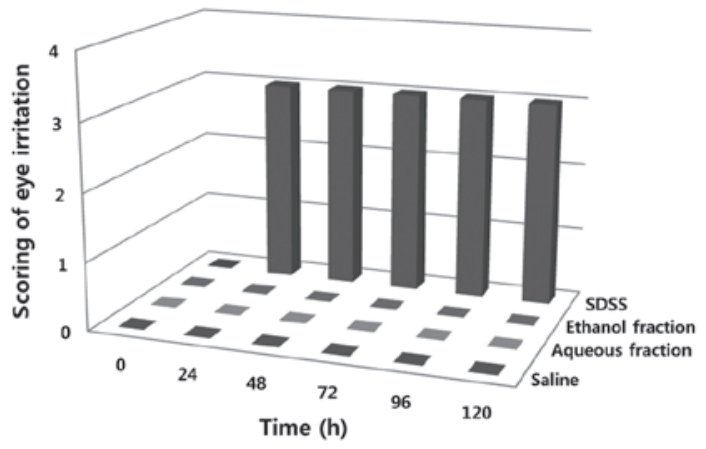

C

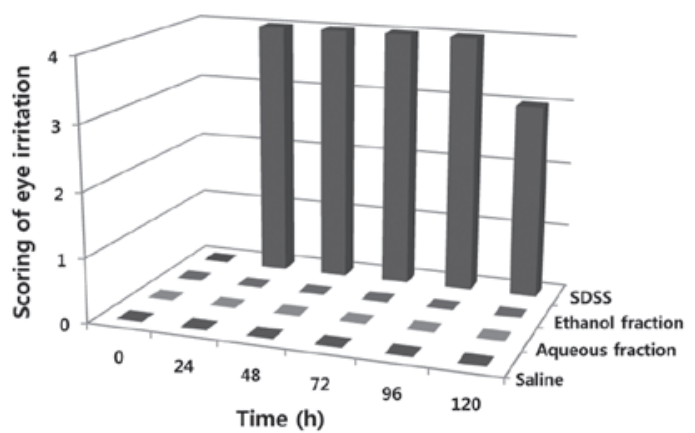

B

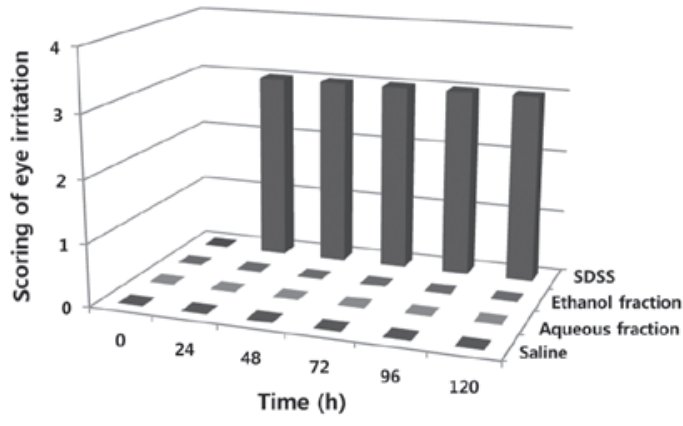

D

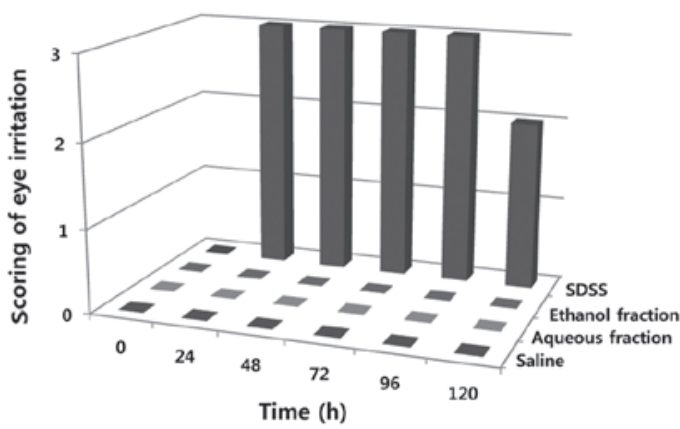

Figure 3. Observation of acute eye irritancy at set time intervals. Scoring standards are similar to those presented in Materials and methods. Observations were made at 6 time-points: 0, 24, 48, 72, 96, and $120 \mathrm{~h}$. The scores of eye irritation are shown at each time interval. (A) Ocular lesions in the cornea, (B) turbid cornea size, (C) eyelid swelling and (D) emission production. Data denote a classical result of five independent observations. Saline, 1st lanes; aqueous fraction, 2nd lanes; ethanol fraction, 3rd lanes; SDSS, 4th lanes. SDSS, sodium dioctyl sulfosuccinate.

comparison purposes. On days 1,2, 3, 4, 5, and 7 following the application of the test fractions, we examined the following criteria of the naked eye: corneal opacity, turbidity range, reaction of the iris and conjunctiva, edema and emission discharge. Irritancy in the eye lens mucosa was evaluated based on redness, irritancy, ocular lesions or inflammation by trained examiners under the authority of a professional from the Center of Laboratory Animal and Care, Kyungpook National University.

\section{Results and Discussion}

In the course of screening natural resources such as food and/ or oriental herb plants for active components exerting antiinflammatory effects in cosmetic application, we observed that aqueous and ethanol fractions of the Angelica keiskei leaf exhibit a potent whitening effect at a concentration of $100 \mu \mathrm{g} / \mathrm{ml}$ with no cytotoxicity in both in vitro and in vivo assays (data not shown).

Recently, Angelica sp. has become a valuable food source in Asia, as these edible plants provide health benefits owing to their antioxidant activities, although some species have a pungent flavor (4). The leaf is consumed by vegetarians both in restaurants and at home and contains various nutrients including vitamins, flavonoids, flavonol and other polyphenol compounds $(9,10)$. For the approval of cosmetics produced using food or chemical sources, the Korean Food and Drug Administration accepts only safety data derived from the most widely used animal tests, such as the Draize eye irritancy test, which involves placing drops of the substance into rabbit eyes. The guidelines for the use of a test compound in cosmetics as a mixture/ingredient involves the control of specific skin toxicity (18). The main constituents associated with toxicity are complicated by the presence of various components such as amine, nitrous compounds and other beneficial and/or disadvantageous substances. Nevertheless, several reports on the antitumor, anti-diabetic and anti-inflammatory activities of known or unknown extracts/fractions of herbal or medicinal plants have been documented thus far $(2,3)$. In previous studies aimed at promoting biological and other applicable purposes, we examined whether these fractions/extracts have biological activity in ameliorating degenerative disorders, including atopic dermatitis. Initially, food ingredients are produced using raw grains, cereals, fruits, vegetables and medicinal herbs and thereafter processed into biomaterials using a range of methods. Following the application of valuable supplementary techniques, such as supercritical extraction, microbial fermentation, biotransformation or chemical modification, biomaterials may be converted into a cosmetic, cosmeceutical, neutraceutical or drug. For this reason, we developed a screening system for antioxidant and anti-tyrosinase agents from medicinal plant extracts and examined their anti-asthmatic activities, as described previously $(19,20)$.

Safety data were obtained following the administration of aqueous and ethanol fractions of the Angelica keiskei leaf (Fig. 1D and E; $100 \mathrm{mg} / \mathrm{ml}$ in a total volume of $100 \mu \mathrm{l}$ ) into rabbit eyes. When saline was used as the control, we did not observe congestion symptoms around the pupil and whites of the eye (data not shown). The Draize eye irritancy test is strictly observational and does not adequately reflect the degree of irritancy in humans, therefore it is generally considered crude, imprecise and unreliable. However, this remains the most convincing test 
in animal models. We applied the following criteria for precise evaluation of toxic symptoms: swelling, inflammation and ulceration on the eye lens. Initially, following treatment with aqueous and ethanol fractions or saline, eyelid and eye mucosa membranes were examined daily. In terms of ocular lesions in the cornea, scores were measured as described in Materials and methods. Lesions were scored using a point system: 0 for no suppuration or haze, 1 when slightly opaque compared to the normal difference in transparency, 2 when easily observed as partly semi-transparent, 3 when not observed at the end of the pupil and 4 when the cornea, but not the iris, was opaque and turbid.

Aqueous and ethanol fractions of Angelica keiskei leaf did not induce perforated ocular lesions in the cornea (Fig. 2, dotted section of column 4), similar to the patterns observed with saline (Fig. 2, column 1) with no additional symptoms. In terms of turbid cornea size, scores were measured as follows: 0 for no turbidity, 1 when the size of the cornea was $1 / 4$ or less, 2 when greater than $1 / 4$ but less than $1 / 2,3$ when greater than $1 / 2$ but less than $3 / 4$ and 4 when greater than $3 / 4$ up to a value of 1 or more. Notably, cornea size and turbidity were not affected by the fractions (Fig. 2, columns 3 and 4). Additionally, the effects of the fractions on eyelid swelling were examined. Eyelid swelling was scored as follows: 0 for no swelling, 1 when normal or slightly swollen (including nictitating membrane), 2 in cases of significant swelling of the eyelid resulting in partial abduction, 3 when swelling of the eyelid was approximately half of the wound and 4 when more than half of the eyelid was swollen. Using the point scoring system, we confirmed that the eyelid was not affected in terms of swelling upon treatment with the fractions (Fig. 2, columns 2 and 3). Production of emissions was analyzed as follows: 0 for no emission, 1 when a small amount of internal eyelash was observed, 2 for wet exhaust and 3 when a large area around the eye and eyelid and/or eyelashes had wet exhaust. As a result, no emission was observed in the eye or eyelid/eyelashes following treatment with the fractions (Fig. 2, columns 2 and 3).

Overall, we detected no changes or damage induced by either the aqueous or ethanol fractions in contrast to sodium dioctyl sulfosuccinate which caused severe toxic symptoms (Fig. 2, comparing column 4 with columns 2 and 3), as assessed by anatomical and clinical observations. The criteria for determining whether or not other parameters are associated with acute eye irritancy were assessed. Observations at the set time intervals (day 1, 2, 3, 4 and 5) revealed that neither the aqueous nor the ethanol fractions affected the ocular lesions in the cornea (Fig. 3A, lanes 2 and 3), turbid cornea size (Fig. 3B, lanes 2 and 3), eyelid swelling (Fig. 3C, lanes 2 and 3) and emission production (Fig. 3D, lanes 2 and 3), whereas sodium dioctyl sulfosuccinate induced perforated damages (Fig. 3, lane 4 of A, B, C and D). Additionally, we did not specify two recommended tests in this report; however, skin irritancy and phototoxicity tests were also negative, whereby wounds treated with the fractions showed similar patterns of recovery as those treated with the control (PBS), and UV exposure of 8-methoxypsoralen plus fraction treatments did not induce tumors in mouse skin (data not shown). A few positive toxicity cases have reportedly been identified from other safety tests, and therefore, we cannot exclude the possibility of toxicity based on acute, sub-acute or chronic safety tests, such as the soap chamber test, repeat insult test or murine local lymph node assay (21-23).

In summary, Angelica keiskei leaf fractions do not induce acute toxicity in the eye irritancy test; specifically, no haze, swelling, redness or emissions in the eye mucosa were observed. Angelica keiskei is therefore a potential candidate for development in the cosmetic industry and/or other applicable purposes. We await the purification and isolation of the pure compound(s), which should aid in establishing those that may be successfully applied for long-term use with no hazardous effects, such as phytoestrogens and toxicants.

\section{Acknowledgements}

This study was supported by the Technology Transfer Project, Kyungpook National University Technopark, Daegu, Korea (S.-H.L). We thank Dr Jin-Chul Heo and Dong-Yoon Nam for their technical assistance. This research was also supported by Kyungpook National University Research Fund, 2012.

\section{References}

1. Sørensen JM: Herb-drug, food-drug, nutrient-drug, and drug-drug interactions: mechanisms involved and their medical implications. J Altern Complement Med 8: 293-308, 2002.

2. Pan MH, Lai CS, Dushenkov S and Ho CT: Modulation of inflammatory genes by natural dietary bioactive compounds. J Agric Food Chem 57: 4467-4477, 2009.

3. Pan MH, Ghai G and Ho CT: Food bioactives, apoptosis, and cancer. Mol Nutr Food Res 52: 43-52, 2008

4. Sarker SD and Nahar L: Natural medicine: the genus Angelica. Curr Med Chem 11: 1479-1500, 2004.

5. Piersen CE: Phytoestrogens in botanical dietary supplements: implications for cancer. Integr Cancer Ther 2: 120-138, 2003.

6. Lopes D, Strobl H. Kolodziejczyk P: 14-Methylpentadecano-15lactone (muscolide): a new macrocyclic lactone from the oil of Angelica keiskei L. Chem Biodivers 1: 1880-1887, 2004.

7. Sigurdsson S, Ogmundsdottir HM and Gudbjarnason S: Antitumour activity of Angelica keiskei leaf extract. In Vivo 19: 191-194, 2005.

8. Sigurdsson S, Ogmundsdottir HM and Gudbjarnason S: The cytotoxic effect of two chemotypes of essential oils from the fruits of Angelica keiskei L. Anticancer Res 25: 1877-1880, 2005.

9. Yeh ML, Liu CF, Huang CF and Huang TC: Hepatoprotective effect of Angelica keiskei in chronically ethanol-treated mice. Pharmacology 68: 70-73, 2003.

10. Heo JC and Lee SH: Amelioration of asthmatic-related symptoms by an aqueous extract of Angelica archangelica L. J Life Sci 18: 1336-1341, 2008

11. Tavaszi J, Budai P, Pálovics A and Kismányoki A: An alternative test battery in detecting ocular irritancy of agrochemicals. Commun Agric Appl Biol Sci 73: 891-895, 2008.

12. Scott L, Eskes C, Hoffmann S, Adriaens E, Alepée N, Bufo M, Clothier R, Facchini D, Faller C, Guest R, et al: A proposed eye irritation testing strategy to reduce and replace in vivo studies using bottom-up and top-down approaches. Toxicol In Vitro 24: $1-9,2010$.

13. Osborne R, Perkins MA and Roberts DA: Development and intralaboratory evaluation of an in vitro human cell-based test to aid ocular irritancy assessments. Fundam Appl Toxicol 28: 139-153, 1995.

14. Nigam PK: Adverse reactions to cosmetics and methods of testing. Indian J Dermatol Venereol Leprol 75: 10-18, 2009.

15. Zimmermann M: Ethical guidelines for investigations of experimental pain in conscious animals. Pain 16: 109-110, 1983.

16. Draize JH, Woodard G and Calvery HO: Methods for the study of irritation and toxicity of substances applied topically to the skin and mucous membranes. J Pharmacol Exp Ther 82: 377-390, 1944.

17. Aoshima H, Saitoh Y, Ito S, Yamana S and Miwa N: Safety evaluation of highly purified fullernenes: based on screening of eye and skin damage. J Toxicol Sci 34: 555-562, 2009. 
18. Draize JH: Dermal toxicity. In: Appraisal of the Safety of Chemicals in Foods, Drugs and Cosmetics. Association of Food and Drug Officials of the United States. Texas State Departmemt of Health, Austin, Texas, pp46-59, 1959.

19. Heo JC, Park JY, Kwon TK, Chung SK, Kim SU and Lee SH: Development of high-throughput screening method for antiasthmatic agents by food-derived libraries. Korean J Food Preserv 12: 267-274, 2005

20. Heo JC, Woo SW, Kweon MA, Park JY, Lee HK, Son M, Rho JR and Lee SH: Aqueous extract of a seed from Helianthus annuus alleviates asthmatic symptoms in vivo. Int J Mol Med 21: 57-61, 2008.

21. Korting HC, Herzinger T, Hartinger A, Kerscher M Angerpointner T and Maibach HI: Discrimination of the irritancy potential of surfactants in vitro by two cytotoxicity assays using normal human keratinocytes, HaCaT cells and 3T3 mouse fibroblasts: correlation with in vivo data from a soap chamber assay. $\mathrm{J}$ Dermatol Sci 7: 119-129, 1994
22. Tardiff RG, Hubner RP and Graves CG: Harmonization of thresholds for primary skin irritation from results of human repeated insult patch tests and laboratory animal skin irritation tests. J Appl Toxicol 23: 279-281, 2003.

23. Basketter DA and Kimber I: Skin irritation, false positives and the local lymph node assay: a guideline issue? Regul Toxicol Pharmacol 61: 137-140, 2011 